1 Novel model to study the physiological effects of temporary or prolonged sex steroid deficiency in male mice

Na Ri Kim ${ }^{1}$, Rougin Khalii ${ }^{1}$, Karel David ${ }^{1}$, Leen Antonio ${ }^{1}$, Dieter Schollaert ${ }^{1}$, Ludo Deboel ${ }^{1}$, Erik Van Herck $^{1}$, Nele Wardenier ${ }^{2}$, Martine Cools ${ }^{2}$, Brigitte Decallonne ${ }^{1}$, Frank Claessens ${ }^{3,5}$, Vanessa Dubois $^{1, \$}$ and Dirk Vanderschueren ${ }^{1, \$, *}$

$6{ }^{1}$ Clinical and Experimental Endocrinology, Department of Chronic Diseases, Metabolism, and Ageing

7 (CHROMETA), KU Leuven, Leuven, Belgium

82 Department of Internal Medicine and Pediatrics, Ghent University and Division of Pediatric 9 Endocrinology, Department of Pediatrics, Ghent University Hospital, Ghent, Belgium

$10{ }^{3}$ Molecular Endocrinology Laboratory, Department of Cellular and Molecular Medicine, KU Leuven, 11 Leuven, Belgium

12 \$Contributed equally as last authors

$13{ }^{*}$ Corresponding author

14

Corresponding author:

Dirk Vanderschueren MD, PhD

17 Clinical and Experimental Endocrinology, KU Leuven

Herestraat 49 box 902

3000 Leuven, Belgium

Tel. +3216346987

dirk.vanderschueren@uzleuven.be

Short running title: Effects of temporary versus prolonged sex steroid deficiency in male mice

Supplemental material is available at

URL: https://figshare.com/s/1bf87d0ecb3dec33de6a

DOI: http://doi.org/ 10.6084/m9.figshare.13303508 
Sex steroids are critical for skeletal development and maturation during puberty as well as skeletal maintenance during adult life. However, the exact time during puberty when sex steroids have the highest impact as well as the ability of bone to recover from transient sex steroid deficiency is unclear. Surgical castration is a common technique to study sex steroid effects in rodents, but it is irreversible, invasive, and associated with metabolic and behavioral alterations. Here, we used a low dose (LD) or a high dose (HD) of gonadotropin-releasing hormone antagonist to either temporarily or persistently suppress sex steroid action in male mice, respectively. The LD group, a model for delayed puberty, did not show changes in linear growth or body composition, but displayed reduced trabecular bone volume during puberty, which fully caught up at adult age. In contrast, the HD group, representing complete pubertal suppression, showed a phenotype reminiscent of that observed in surgically castrated rodents. Indeed, HD animals exhibited severely impaired cortical and trabecular bone acquisition, decreased body weight and lean mass, and increased fat mass. In conclusion, we developed a rodent model of chemical castration, which can be used as an alternative to surgical castration. Moreover, the transient nature of the intervention enables to study the effects of delayed puberty and reversibility of sex steroid deficiency.

Key words: delayed puberty, bone, body composition, hypogonadotropic hypogonadism 


\section{NEW \& NOTEWORTHY}

48 We developed a rodent model of chemical castration, which can be used as an alternative to surgical 49 castration. Moreover, the transient nature of the intervention enables to study the effects of delayed 50 puberty and reversibility of sex steroid deficiency.

51 


\section{INTRODUCTION}

Puberty is a critical period not only for the maturation of the reproductive system, but also for skeletal development and maturation ${ }^{35}$. Rodent studies have confirmed the pivotal role of sex steroids for bone mass acquisition and growth during puberty, as illustrated by the severely reduced cortical as well as trabecular bone mass in adult male mice as a result of surgical castration in early puberty ${ }^{7}$. However, it is not clear to what extent the decrease in bone mass due to pubertal sex steroid deprivation is reversible. The plasticity of bone as well as the timing of sex steroid action during puberty are important open questions in bone physiology, in particular, in the context of a delayed pubertal onset.

Delayed puberty is defined as a lack of development of sexual characteristics by an age equaling two standard deviations beyond the population mean. It is a common condition, particularly in boys, with a prevalence of 1 in 50 individuals ${ }^{18}$. The impact of a delayed pubertal onset on bone health remains controversial. Indeed, according to some studies, boys suffering from delayed puberty fail to achieve optimal peak bone mass acquisition ${ }^{9,14,15,27}$, while other studies suggest that these individuals eventually catch up and obtain their full genetic height potential as well as volumetric bone mineral density (BMD) in spite of the delay of their growth spurt ${ }^{8,24,26,36}$. This controversy may partly be explained by a latency between the diagnosis of this condition and the study of its impact on bone health later in life since bone mass acquisition in humans is only optimal at the end of the second decade of life $e^{2,3,5}$. This illustrates the need for a suitable animal model of reversible sex steroid deficiency early in puberty.

Surgical castration is a common technique to study the effect of sex steroid deficiency in rodent models. However, this approach is not only invasive, but also irreversible which does not allow to explore the timing of sex steroid action, especially during puberty. Moreover, it has no equivalent in clinical practice, where chemical castration using gonadotropin-releasing hormone (GnRH) analogues is applied in certain conditions, for example for gonadal steroid suppression in adolescents presenting precocious puberty or for androgen deprivation in prostate cancer patients ${ }^{31,36}$. These compounds block (GnRH antagonists) or desensitize (GnRH agonists) the GnRH receptors in the pituitary gland, thereby suppressing luteinizing hormone (LH) secretion and eventually reducing production and secretion of testosterone $(T)$. However, the effects of chemical castration on bone, growth and body composition have not been clearly characterized in rodents.

The aim of our study was therefore twofold: first, to establish a mouse model of temporary sex steroid deficiency during puberty and investigate the impact on bone, growth and body composition at adult age. Second, to study the impact of prolonged sex steroid deficiency induced by chemical castration on bone, growth and body composition in mice. To do so, we optimized the dosage of a $\mathrm{GnRH}$ analogue to obtain reversible short-term versus prolonged long-term chemical castration in a rodent model. We hypothesized that the use of a low dose of GnRH analogue administered at the start of puberty would be able to induce transient sex steroid deficiency, allowing to study the timing of sex steroid action as well as introducing a novel animal model of delayed puberty. In addition, a high dose of $\mathrm{GnRH}$ analogue would be suitable for complete and persistent suppression of pubertal sex steroid secretion ${ }^{33}$, allowing to assess the effects of chemical castration - as opposed to surgical castration - on bone and body composition. As a GnRH analogue, we decided to use a GnRH antagonist because this does not cause an initial flare-up response by means of a surge in LH levels as observed with GnRH agonists ${ }^{16,19}$. 


\section{MATERIALS AND METHODS}

\section{Animal experiments}

In a pilot study, male wild type C57BL/6J mice (Charles River, MA, USA) were randomized into five groups, each consisting of 3 animals/group. Single injections of different doses of degarelix (DGX) (Ferring Pharmaceuticals, Saint-Prex, Switzerland), a gonadotropin-releasing hormone (GnRH) antagonist, were used to determine the optimal dose needed to obtain a delayed onset of puberty. Injections at 4 weeks of age of either sterile water as vehicle control or DGX $(0.2,0.5,1$, or $2 \mathrm{mg} / \mathrm{kg})$ were given subcutaneously. Two and four weeks after DGX administration (i.e. at 6 and 8 weeks of age, respectively), animals were sacrificed and seminal vesicle weight was used as a readout for sex steroid activity. Based on the seminal vesicle weights at 6 weeks of age, we conducted a power analysis which indicated that to detect a difference between the vehicle control and the $2 \mathrm{mg} / \mathrm{kg}$ DGX groups (effect size 1.78) with a power of $90 \%$, a sample size of at least 8 animals per group is required. Therefore, additional animals ( $n=5 /$ group) were injected with vehicle control or $2 \mathrm{mg} / \mathrm{kg}$ DGX at 4 weeks of age. Two weeks after DGX administration (i.e. at 6 weeks of age), nose-to-tail length and body composition were determined and tissues were collected for bone and serum analysis.

In a next experiment, male wild type C57BL/6J mice (Charles River, MA, USA) were randomly assigned to one of the three following groups; (1) control (C) (n=8), (2) a low dose of DGX (LD) to mimic delayed puberty $(n=10),(3)$ a high dose of DGX (HD) to model complete suppression of puberty $(n=11)$. At 4 weeks of age, mice were subcutaneously injected with vehicle (C), $2 \mathrm{mg} / \mathrm{kg}$ DGX (LD) or $25 \mathrm{mg} / \mathrm{kg}$ DGX (HD). Mice of the HD group were additionally injected with $25 \mathrm{mg} / \mathrm{kg}$ of DGX at 8 and 12 weeks of age, while mice of the C and LD groups received vehicle at those time points. Anogenital distance and body weight were monitored weekly from 4 to 16 weeks. Animals were euthanized at 16 weeks (adult age) for assessment of growth, body composition, bone parameters, and sex steroid-sensitive tissue weights. Nose-to-tail length was measured before euthanasia in anesthetized animals using a ruler. In a separate experiment, the HD protocol was applied to female wild type C57BL/6J mice (KU Leuven animal facility, Belgium), which were injected with either vehicle control $(n=6)$ or $25 \mathrm{mg} / \mathrm{kg} \mathrm{DGX} \mathrm{(n=6)}$ at 4,8 , and 12 weeks of age and euthanized at 16 weeks of age. At termination point, uterus weight was determined as a readout for circulating sex steroids.

Mice were group-housed (3-5 animals/cage) in conventional facilities at $20^{\circ} \mathrm{C}$ with 12 -hour light/dark cycle and ad libitum access to water and standard chow, according to our institutional guidelines. All animals were euthanized by sodium pentobarbital overdose (Dolethal, Vétoquinol Ltd, Buckingham, UK) (intraperitoneal injection of $74 \mathrm{mg} / \mathrm{kg}$ ) followed by cardiac puncture. All animal experiments were approved by the KU Leuven ethical committee (P192/2016).

\section{Micro-computed tomography}

Both axial and appendicular bones were scanned using Skyscan 1172 (Bruker, Kontich, Belgium) with $5 \mu \mathrm{m}$ pixel size, $0.5 \mathrm{~mm}$ Al filter, $50 \mathrm{kV}, 200 \mu \mathrm{A}, 180^{\circ}$ angular rotation at $0.4^{\circ}$ steps, and $590 \mathrm{~ms}$ integration time. All images were reconstructed using the NRecon program and analyzed by CTAn software as previously described ${ }^{28}$. For cortical bone, a $0.5 \mathrm{~mm}$ region of interest in the distal femur was selected starting at $4.5 \mathrm{~mm}$ from the distal edge of the growth plate. For femoral trabecular bone, a $2 \mathrm{~mm}$ segment starting $0.25 \mathrm{~mm}$ from distal growth plates was analyzed. For trabecular bone of vertebrae, the whole vertebral body of lumbar 5 (L5) was analyzed. Parameters included trabecular bone volume fraction (BV/TV, \%), trabecular number (Tb.N, 1/mm), trabecular thickness (Tb.Th, $\mu \mathrm{m})$, trabecular separation (Tb.Sp, $\mu \mathrm{m})$, total cross-sectional tissue area (Tt.Ar, $\mathrm{mm}^{2}$ ), cortical bone area $\left(\mathrm{Ct} . \mathrm{Ar}, \mathrm{mm}^{2}\right)$, medullary area $\left(\mathrm{Ma} . \mathrm{Ar}, \mathrm{mm}^{2}\right)$, cortical thickness $(\mathrm{Ct} . \mathrm{Th}, \mathrm{mm})$, periosteal circumference (Ct.PC, mm), endosteal circumference (Ct.EC, $\mathrm{mm}$ ), and polar moment of inertia (J, $\mathrm{mm}^{4}$ ).

\section{Body composition}


Body composition was measured by quantitative magnetic resonance (EchoMRI -100H Analyzer; Echo

144 Medical Systems, Houston, TX, USA).

Serum analysis

147 Serum levels of T were measured in a single run by a two-dimensional liquid chromatography system 148 and an AB/Sciex QTrap 5500 tandem mass spectrometer in atmospheric pressure chemical ionization positive $(A P C I)$ mode $^{21}$. Serum IGFBP-3 levels were measured using a commercial enzyme-linked immunosorbent assay kit (RAB0236, Sigma-Aldrich) according to the manufacturers' instructions. Serum IGF-1 and osteocalcin levels were measured using an in-house radioimmunoassay (RIA) as 152 previously described ${ }^{40}$.

\section{Statistics}

154 Statistical analysis was performed using GraphPad Prism v7.04 (GraphPad, La Jolla, CA, USA). Unpaired two-tailed student's $t$-test and one-way ANOVA with Bonferroni post-hoc test were used to analyze differences between two or more groups, respectively. Two-way ANOVA with Bonferroni post-hoc test was used in experiments with more than one independent variable. Data are represented as mean $\pm \mathrm{SEM}$, and $\mathrm{p}<0.05$ was considered as statistically significant. 


\section{RESULTS}

\section{Dose-dependent and transient effect of GnRH antagonist administration on sex steroid suppression}

In order to determine the optimal dose of the GnRH antagonist degarelix (DGX) that induces transient sex steroid deficiency during puberty, we performed a pilot experiment in which a single administration of a variable dose of DGX was tested in prepubertal wild type male mice. At 4 weeks of age, mice were subcutaneously injected with either sterile water as vehicle control or DGX $(0.2$, $0.5,1$, or $2 \mathrm{mg} / \mathrm{kg}$ ). The weight of seminal vesicles (SV) is commonly used as a proxy for systemic sex steroid activity ${ }^{7}$. While body weight was not different between the various groups of DGX-treated mice compared to control (Fig. 1A), a dose-dependent reduction in SV weight was observed (Fig. 1B). Indeed, 2 weeks after single injection, both 1 and $2 \mathrm{mg} / \mathrm{kg}$ DGX groups showed significant reduction in SV weight compared to control, with strongest reduction in the $2 \mathrm{mg} / \mathrm{kg}$ group $(-36.6 \%,-77.3 \%)$. Reduction in SV weight was still present in both groups 4 weeks after single DGX injection but to a lesser extent $(-23.8 \%,-25.6 \%)$, indicating recovery from sex steroid suppression (Fig. 1B). Based on these findings, we chose $2 \mathrm{mg} / \mathrm{kg}$ as the low DGX dose (LD) to mimic a delay in puberty and compared this group with vehicle-treated control mice (C) as well as with mice receiving a $25 \mathrm{mg} / \mathrm{kg}$ high DGX dose (HD) to model complete and persistent suppression of puberty ${ }^{33}$.

Effect of delayed puberty and complete pubertal suppression on body composition and sex steroid action

We measured anogenital distance (AGD) as a biomarker to evaluate sex steroid action ${ }^{34}$. Consistent with a delay in puberty, the LD group showed a significant decrease in AGD at 5 and 6 weeks of age compared to the control group, while the difference in AGD between these two groups disappeared by the end of puberty (week 8), indicating full recovery of sex steroid action (Fig. 2A). On the other hand, the HD group displayed significant reduction in AGD at all time points compared to the control group, in line with puberty being completely suppressed (Fig. 2A). Body weight as well as body composition were comparable between control and LD groups during puberty and at adult age, except for a small but significant decrease in lean mass in LD animals at 6 weeks of age, which recovered at 16 weeks (Fig. 2B-D). However, the HD group showed reduced body weight compared to control during the entire time course of the experiment (Fig. 2B), in line with the effect of prepubertal surgical castration on body weight ${ }^{12}$. Moreover, HD animals displayed a shift in body composition at adult age, with fat mass being increased (+6.9\%) along with a decrease in lean mass (9.0\%) (Fig. 2D). Body weight gain (BWG) was highest during early puberty (before 6 weeks of age ${ }^{7}$ ) indicating a growth spurt in this period (Fig. 2E). However, there were no differences in BWG between the three groups in early puberty (Fig. 2E). In contrast, in late puberty ( 6 to 8 weeks of age ${ }^{7}$ ), the LD group showed a significant higher BWG $(+2.3 \%)$ than the control group, while the HD group exhibited a lower BWG compared to the control (-1.7\%) and LD (-4.0\%) groups (Fig. 2E). Also in young adulthood (9 to 12 weeks of age), BWG was lower in HD compared to control (-1.7\%) and LD (-2.5\%) groups (Fig. 2E). At 16 weeks of age, there was no difference between control and LD groups in circulating T levels (Fig. 2F) or sex steroid-sensitive tissue weights, namely levator ani muscle, testes, and SV (Fig. 2G-I), consistent with a full recovery of sex steroid function. In contrast, the HD group showed robust reduction of these parameters (Fig. 2F-I), indicating strong and persistent sex steroid suppression. In particular, SV weight of the HD group was similar to that of surgically castrated mice (Fig. 2l, right panel). When applying the HD protocol to female mice, a drastic reduction in uterus weight was observed at 16 weeks of age (Supplemental Fig. S1; Supplemental material is available at https://figshare.com/s/1bf87d0ecb3dec33de6a).

Effect of delayed puberty and complete pubertal suppression on bone mass acquisition and growth

To assess the effect of delayed puberty on bone in mid-puberty and at adult age, femoral cortical and trabecular bone were analyzed. At 6 weeks of age (mid-puberty), there were no differences in cortical bone parameters, including cortical bone thickness, in the LD group compared to control (Fig. 3A-D). However, trabecular bone volume was significantly decreased (Fig. 3E). Reduced trabecular 
bone volume was accompanied by increase in trabecular separation and decrease in trabecular number but not thickness (Fig. 3F-H). As a biochemical marker of bone turnover ${ }^{10}$, serum osteocalcin was measured. In line with the decrease in trabecular bone volume, serum osteocalcin tended to increase in LD group compared to control at 6 weeks of age (Fig. 3I). Since puberty is also a critical period for linear growth ${ }^{7}$, body and bone (appendicular and axial) length were measured as well at 6 weeks. There were no differences in nose-to-tail, femur, and lumbar 5 (L5) column length between LD and control groups (Fig. 4A-C). Circulating serum insulin like growth factor-1 (IGF-1) and insulin like growth factor binding protein-3 (IGFBP-3) levels, as a proxy for growth hormone action, were also unaffected in LD animals at 6 weeks (Fig. 4D, E)

At 16 weeks (adult age), the decrease in trabecular bone volume was no longer observed in the LD group (Fig. 5A-D and Table 1), indicating that the deleterious effects on bone observed during delayed puberty fully disappear at adult age. The HD group, on the other hand, exhibited both cortical and trabecular bone loss at 16 weeks (Fig. 5A-D and Table 1), consistent with strong and persistent sex steroid suppression ${ }^{38}$. In line with the bone loss, the HD group showed lower polar moment of inertia, which is a proxy for bone strength, and increased serum osteocalcin levels compared to the control group, while these parameters were unaffected in LD animals (Fig. 5E-F). Overall, LD had no major effects on body and bone length at 16 weeks, except for a significant but small increase in L5 column length (Fig. 6A-C). In the HD group, continuous strong sex steroid suppression resulted in increased nose-to-tail length at adult age, which was accompanied by a trend towards increased femur and column length (Fig. $6 \mathrm{~A}-\mathrm{C}$ ) as well as significant increase in serum IGF-1 and IGFBP-3 levels (Fig. 6D, E). 
232 Sex steroids are critical for development and maturation of several organs, including the reproductive and musculoskeletal system, in particular, during puberty. Surgical castration is a common technique to study the effects of sex steroid deprivation in rodent models. However, this approach is irreversible and hence does not allow to study timing of sex steroid action. In addition, abdominal surgery has been associated with changes in food and water consumption as well as behavioral alterations, which may confound the observations ${ }^{20}$. In the present study, summarized in Fig. 7, we used a low dose of GnRH antagonist to established an animal model for temporary sex steroid deficiency in male mice, enabling to study timing and reversibility of sex steroid action. In addition, a high dose of GnRH antagonist induced complete and persistent suppression of sex steroid action. Our chemical castration model can therefore be used as an alternative to surgical castration for the study of sex steroid effects in rodents, avoiding confounding effects from surgery.

Induction of temporary sex steroid deficiency using single prepubertal injection of a low dose of GnRH antagonist did not affect growth or body composition at adult age (Fig.7, left panel). However, delayed pubertal onset was accompanied by reduced trabecular bone volume during puberty, which fully recovered at adult age. This finding has two major implications. First, it confirms the high sensitivity of trabecular bone to circulating sex steroids ${ }^{6}$ and illustrates the plasticity of bone, which is able to recover from transient sex steroid deprivation. Second, it sheds light onto the clinical controversy regarding the effect of delayed puberty on bone health at maturity ${ }^{17,43}$. In some studies, boys with delayed puberty had lower volumetric BMD and bone mass ${ }^{24,26}$. Other studies, however, reported that they showed normal volumetric $\mathrm{BMD}^{4,42}$ and serum bone turnover markers which were similar to healthy children ${ }^{25}$. Our work suggests that, even if delayed pubertal timing is associated with bone loss during puberty, this deleterious effect does not persist at adult age. Hence, these findings are in support of a watchful waiting approach in the clinical context of delayed puberty ${ }^{43}$.

In contrast to the reversible effects of transient sex steroid deficiency on bone, male mice with persistent sex steroid deficiency due to complete pubertal suppression showed reduction of both cortical and trabecular bone mass at adult age (Fig. 7, right panel). Also, these mice exhibited a decrease in body weight accompanied by increased and decreased fat and lean body mass, respectively. These findings are in line with observations in surgically castrated animals. Indeed, prepubertal orchidectomy of male mice resulted in decreased body weight at adult age ${ }^{12,39}$, while limiting cortical radial bone development and leading to a reduction of cortical as well as trabecular bone acquistion ${ }^{7,39}$. The shift in body composition towards increased fat mass and decreased lean mass is also in line with reports in surgically castrated rodents ${ }^{22,23,37}$, although in some studies orchidectomy needed to be combined with high fat diet to observe this shift ${ }^{12}$. In contrast to surgical castration which has no effect on body or bone length ${ }^{41}$, we observed an increased linear growth (body and appendicular bone length) as well as elevated circulating IGF-1 levels in our model of persistent sex steroid deficiency induced by chemical castration. Given the complexity of the crosstalk between sex steroids and the growth hormone/IGF-1 axis ${ }^{1}$, further investigation is however required to determine whether the elevated IGF-1 is causal for the increased linear growth of the HD animals. Interestingly, the finding of increased linear growth is reminiscent of the clinical observation in patients with hypogonadotropic hypogonadism. Indeed, in these patients, growth continues until the third decade leading to increased final height ${ }^{32}$. Also, in line with our mouse model, these patients show low cortical bone mass due to absence of sex steroid action on bone during puberty $^{13,30}$ as well as increased adiposity ${ }^{11}$.

In summary, our study demonstrates that a high dose of GnRH antagonist induces complete and prolonged sex steroid suppression in mice, providing an alternative method to surgical castration in 
277 animal studies. In addition, using a low dose of GnRH antagonist, we developed the first animal 278 model that enables to explore timing and reversibility of sex steroid action. The main limitation of 279 our study is that the effects of transient and persistent sex steroid deficiency were mainly investigated in male mice. Although the drastic reduction in uterus weight suggests that our protocol might also be suitable to induce sex steroid deprivation in female mice, additional investigation is needed to fully characterize the effects on female growth, body composition and bone. In addition, there are differences in sex steroid physiology between humans and mice ${ }^{29}$ which might limit the clinical translation of our findings. Nevertheless, our model provides future opportunities to study the timing and reversibility of sex steroid action on different aspects of physiology, such as sexual function, physical activity, cognition, behavior, and ageing. 
289 Degarelix was a kind gift from Ferring Pharmaceuticals.

290 GRANTS

291 This work was funded by a research grant from the Flemish Fund for Scientific Research (FWO; 292 GOD2217N).

293 DISCLOSURES

294 The authors declare no competing interests.

\section{AUTHOR CONTRIBUTIONS}

296 Study design: D.V., V.D., F.C., and B.D. Study conduct: N.R.K., D.S., L.D., and E.V.H. Data collection: 297 N.R.K. Data analysis: N.R.K. Data interpretation: N.R.K., L.A., B.D., F.C., V.D., and D.V. Drafting 298 manuscript: N.R.K. and V.D. Revising manuscript content: all authors. Approving final version of 299 manuscript: all authors.

300 


\section{REFERENCES}

1. Almeida, M. et al. Estrogens and androgens in skeletal physiology and pathophysiology. Physiol. Rev. (2017). doi:10.1152/physrev.00033.2015

2. Bailey, D. A., Faulkner, R. A. \& Mc kay, H. A. Growth, physical activity, and bone mineral acquisition. Exerc. Sport Sci. Rev. (1996). doi:10.1249/00003677-199600240-00010

3. Bailey, D. A., McKay, H. A., Mirwald, R. L., Crocker, P. R. E. \& Faulkner, R. A. A six-year longitudinal study of the relationship of physical activity to bone mineral accrual in growing children: The University of Saskatchewan Bone Mineral Accrual Study. J. Bone Miner. Res. (1999). doi:10.1359/jbmr.1999.14.10.1672

4. Bertelloni, S., Baroncelli, G. I., Ferdeghini, M., Perri, G. \& Saggese, G. Normal volumetric bone mineral density and bone turnover in young men with histories of constitutional delay of puberty. J. Clin. Endocrinol. Metab. (1998). doi:10.1210/jcem.83.12.5348

5. Bonjour, J. Bone mineral acquisition in adolescence. (Academic Press, 1996).

6. Callewaert, F. et al. Differential regulation of bone and body composition in male mice with combined inactivation of androgen and estrogen receptor- $\alpha$. FASEB J. (2009). doi:10.1096/fj.08-113456

7. Callewaert, F. et al. Sexual dimorphism in cortical bone size and strength but not density is determined by independent and time-specific actions of sex steroids and IGF-1: Evidence from pubertal mouse models. J. Bone Miner. Res. (2010). doi:10.1359/jbmr.090828

8. Cools, B. L. M., Rooman, R., Op De Beeck, L. \& Du Caju, M. V. L. Boys with a simple delayed puberty reach their target height. Horm. Res. (2008). doi:10.1159/000137663

9. Crowne, E. C., Shalet, S. M., Wallace, W. H. B., Eminson, D. M. \& Price, D. A. Final height in boys with untreated constitutional delay in growth and puberty. Arch. Dis. Child. (1990). doi:10.1136/adc.65.10.1109

10. Delmas, P. D. Biochemical markers of bone turnover. J. Bone Miner. Res. (1993). doi:10.1002/jbmr.5650081323

11. Dhindsa, S., Ghanim, H., Batra, M. \& Dandona, P. Hypogonadotropic hypogonadism in men with diabesity. Diabetes Care (2018). doi:10.2337/dc17-2510

12. Dubois, V. et al. Androgen deficiency exacerbates high-fat diet-induced metabolic alterations in male mice. Endocrinology (2016). doi:10.1210/en.2015-1713

13. Finkelstein, J. S. et al. Osteoporosis in men with idiopathic hypogonadotropic hypogonadism. Ann. Intern. Med. (1987). doi:10.7326/0003-4819-106-3-

14. Finkelstein, J. S., Klibanski, A. \& Neer, R. M. A longitudinal evaluation of bone mineral density in adult men with histories of delayed puberty. J. Clin. Endocrinol. Metab. (1996). doi:10.1210/jc.81.3.1152

15. Finkelstein, J. S., Neer, R. M., Biller, B. M. k., Klibanski, A. \& Crawford, J. D. Osteopenia in men with a history of delayed puberty. N. Engl. J. Med. (1992). doi:10.1056/NEJM199202273260904

16. Heidenreich, a et al. Guidelines on Prostate Cancer. Update (2011).

17. Howard, S. R. \& Dunkel, L. Delayed Puberty-Phenotypic Diversity, Molecular Genetic Mechanisms, and Recent Discoveries. Endocr. Rev. (2019). doi:10.1210/er.2018-00248

18. Howard, S. R. \& Dunkel, L. The Genetic Basis of Delayed Puberty. Neuroendocrinology (2018). doi:10.1159/000481569

19. Inoue, T. et al. Recovery of Serum Testosterone Levels and Sexual Function in Patients Treated With Short-term Luteinizing Hormone-releasing Hormone Antagonist as a Neoadjuvant Therapy Before External Radiotherapy for Intermediate-risk Prostate Cancer: Preliminary Prospec. Clin. Genitourin. Cancer (2018). doi:10.1016/j.clgc.2017.09.009 
20. Jacobsen, K. R., Kalliokoski, O., Teilmann, A. C., Hau, J. \& Abelson, K. S. P. Postsurgical food and water consumption, fecal corticosterone metabolites, and behavior assessment as noninvasive measures of pain in vasectomized BALB/c Mice. J. Am. Assoc. Lab. Anim. Sci. (2012).

21. Jardí, F. et al. Androgen Receptor in Neurons Slows Age-Related Cortical Thinning in Male Mice. J. Bone Miner. Res. (2019). doi:10.1002/jbmr.3625

22. Jardí, F. et al. Testosterone boosts physical activity in male mice via dopaminergic pathways. Sci. Rep. (2018). doi:10.1038/s41598-017-19104-0

23. Ke, H. Z. et al. Long-term effects of aging and orchidectomy on bone and body composition in rapidly growing male rats. J. Musculoskelet. Neuronal Interact. (2001).

24. Kindblom, J. M. et al. Pubertal timing predicts previous fractures and BMD in young adult men: The GOOD study. J. Bone Miner. Res. (2006). doi:10.1359/jbmr.020602

25. Krupa, B. \& Miazgowski, T. Brief report: Bone mineral density and markers of bone turnover in boys with constitutional delay of growth and puberty. J. Clin. Endocrinol. Metab. 90, 28282830 (2005).

26. Kuh, D. et al. Pubertal timing and bone phenotype in early old age: findings from a British birth cohort study. Int. J. Epidemiol. (2016). doi:10.1093/ije/dyw131

27. LaFranchi, S., Hanna, C. E. \& Mandel, S. H. Constitutional delay of growth: Expected versus final adult height. Pediatrics (1991).

28. Laperre, K. et al. Development of micro-CT protocols for in vivo follow-up of mouse bone architecture without major radiation side effects. Bone (2011). doi:10.1016/j.bone.2011.06.031

29. Laurent, M. R. et al. Sex hormone-binding globulin regulation of androgen bioactivity in vivo: Validation of the free hormone hypothesis. Sci. Rep. (2016). doi:10.1038/srep35539

30. Loro, M. L. et al. Early identification of children predisposed to low peak bone mass and osteoporosis later in life. J. Clin. Endocrinol. Metab. (2000). doi:10.1210/jc.85.10.3908

31. Muller, J., Juul, A., Andersson, A. M., Sehested, A. \& Skakkebaek, N. E. Hormonal changes during $\mathrm{GnRH}$ analogue therapy in children with central precocious puberty. in Journal of Pediatric Endocrinology and Metabolism (2000). doi:10.1515/jpem.2000.13.s1.739

32. Perry, R. J., Farquharson, C. \& Ahmed, S. F. The role of sex steroids in controlling pubertal growth. Clinical Endocrinology (2008). doi:10.1111/j.1365-2265.2007.02960.x

33. Poulsen, C. B., Mortensen, M. B., Koechling, W., Sørensen, C. B. \& Bentzon, J. F. Differences in hypercholesterolemia and atherogenesis induced by common androgen deprivation therapies in male mice. J. Am. Heart Assoc. (2016). doi:10.1161/JAHA.115.002800

34. Schwartz, C. L. et al. Anogenital distance as a toxicological or clinical marker for fetal androgen action and risk for reproductive disorders. Archives of Toxicology (2019). doi:10.1007/s00204-018-2350-5

35. Seeman, E. Sexual Dimorphism in Skeletal Size, Density, and Strength. J. Clin. Endocrinol. Metab. (2001). doi:10.1210/jcem.86.10.7960

36. Shore, N. D., Abrahamsson, P. A., Anderson, J., Crawford, E. D. \& Lange, P. New considerations for ADT in advanced prostate cancer and the emerging role of GnRH antagonists. Prostate Cancer and Prostatic Diseases (2013). doi:10.1038/pcan.2012.25

37. Vandenput, L. et al. Evidence from the aged orchidectomized male rat model that $17 \beta$ estradiol is a more effective bone-sparing and anabolic agent than $5 \alpha$-dihydrotestosterone. J. Bone Miner. Res. (2002). doi:10.1359/jbmr.2002.17.11.2080

38. Vanderschueren, D. et al. Sex steroid actions in male bone. Endocrine Reviews (2014). doi:10.1210/er.2014-1024 
39. Venken, K. et al. Relative impact of androgen and estrogen receptor activation in the effects of androgens on trabecular and cortical bone in growing male mice: A study in the androgen receptor knockout mouse model. J. Bone Miner. Res. (2006). doi:10.1359/jbmr.060103

40. Verhaeghe, J. et al. Bone and mineral metabolism in BB rats with long-term diabetes: Decreased bone turnover and osteoporosis. Diabetes (1990). doi:10.2337/diab.39.4.477

41. Wu, J. et al. Enzalutamide reduces the bone mass in the axial but not the appendicular skeleton in male mice. Endocrinology (2016). doi:10.1210/en.2015-1566

42. Yap, F. et al. The skeletal phenotype of men with previous constitutional delay of puberty. J. Clin. Endocrinol. Metab. (2004). doi:10.1210/jc.2004-0046

43. Zhu, J. \& Chan, Y. M. Adult consequences of self-limited delayed puberty. Pediatrics (2017). doi:10.1542/peds.2016-3177 
Figure 1. Optimization of the delayed puberty model. (A) Body weight evolution during puberty in male WT mice treated with the indicated DGX dose by a single subcutaneous injection at 4 weeks of age ( $n=3 /$ group at each time point). (B) Seminal vesicle wet weight at 6 and 8 weeks of age ( $n=3 /$ group at each time point). Data in A were analyzed with two-way ANOVA and those in B with one-way ANOVA, both with Bonferroni post-hoc test. ${ }^{*} \mathrm{p}<0.05,{ }^{* * *} \mathrm{p}<0.001,{ }^{* * * *} \mathrm{p}<0.0001$ vs. Control mice. $\mathrm{BW}=$ body weight; $\mathrm{DGX}=$ degarelix; SV = seminal vesicle. In all figures, data obtained during puberty are depicted against a green background, while a white background is used for data obtained at adult age.

Figure 2. Effects of delayed onset or complete suppression of puberty on growth and androgensensitive tissues. (A-B) Anogenital distance (A) and body weight (B) from 4 to 16 weeks of age of male WT mice from the indicated groups (number of animals: $C=8, L D=10, H D=11$ ). See text for details about DGX administration. (C-D) Body fat and lean mass measured at 6 (C) and 16 (B) weeks of age (number of animals at 6 weeks: $C=7, L D=8$; at 16 weeks: $C=8, L D=10, H D=11$ ). (E) Body weight gain expressed as the percentage increase per week from 4 to 16 weeks of age of male WT mice from the indicated groups (number of animals: $C=8, L D=10, H D=11$ ). (F) Serum T levels of the mice at 16 weeks of age (number of animals: $C=8, L D=10, H D=11$ ). (G-I) Weight of levator ani muscle $(G)$, testes $(H)$, and seminal vesicles (I left panel) of the mice at 16 weeks of age (number of animals: $C=8, L D=10, H D=11$ ). In I right panel, the seminal vesicle weight of 12 -week-old male WT mice which were sham-operated (SHAM) or surgically castrated (ORX) at 3 weeks of age is indicated for comparison ( $n=7 /$ group). Data in $A, B$, and $E$ were analyzed with two-way ANOVA and data in $D, F$, $\mathrm{G}, \mathrm{H}$ and I left panel with one-way ANOVA, all with Bonferroni post-hoc test. $\mathrm{C}$ and I right panel were analyzed using unpaired two-tailed $t$-test. ${ }^{*} p<0.05,{ }^{* * *} p<0.001,{ }^{* * * *} p<0.0001$ comparison between low dose (LD) and Control (C) or high dose (HD), as indicated. ${ }^{a} p<0.0001,{ }^{b} p<0.001,{ }^{c} p<0.01$ comparison between high dose (HD) and Control (C). AGD = anogenital distance; $B W=$ body weight; $B W G=$ body weight gain; $C$ = control; $\mathrm{HD}=$ high dose; $\mathrm{LA}=$ levator ani; $\mathrm{LD}=$ low dose; $\mathrm{ORX}=$ orchidectomized; $\mathrm{SV}$ $=$ seminal vesicles.

Figure 3. Effects of delayed puberty on bone during puberty. Cortical (A-D) and trabecular (E-H) bone parameters at 6 weeks of age of male WT mice from the indicated groups (number of animals: $C=7, L D=8$ ). See text for details about DGX administration. (I) Serum osteocalcin at 6 weeks of age (number of animals: $C=6, L D=8$ ). Data were analyzed with unpaired two-tailed $t$-test. ${ }^{* *} p<0.01$, ${ }^{* * *} \mathrm{p}<0.001$ vs. Control (C). Ct. Ar = cortical area; $\mathrm{C}=$ control; BV/TV = bone volume fraction; Cort. Th = cortical thickness; $\mathrm{LD}=$ low dose; $\mathrm{Ma}$. Ar = medullary area; $\mathrm{OC}=$ osteocalcin; $\mathrm{Tb} . \mathrm{N}=$ trabecular number; $\mathrm{Tb} . \mathrm{Sp}=$ trabecular separation; $\mathrm{Tb} . \mathrm{Th}=$ trabecular thickness; $\mathrm{Tt}$. Ar = total cross-sectional tissue area.

Figure 4. Effect of delayed puberty on linear growth during puberty. (A-C) Nose-to-tail (A), femur (B), and vertebral L5 column (C) length of 6-week-old male WT mice from the indicated groups (number of animals: $C=5-7, L D=5-8$ ). See text for details about DGX administration. (D-E) Serum levels of IGF-1 (D) and IGFBP-3 (E) in 6-week-old mice (number of animals: $C=6, L D=8$ ). Data were analyzed with unpaired two-tailed $t$-test. $C=$ control; IGF-1 = insulin like growth factor-1; IGFBP-3 = insulin like growth factor binding protein-3; $L 5=$ lumbar $5, L D=$ low dose.

Figure 5. Effects of delayed onset or complete suppression of puberty on bone at adult age. (A-B) 3D micro-computed tomography images of the femoral cortical (A) and trabecular (B) bone at 16 weeks of age of male WT mice from the indicated groups (number of animals: $C=8, L D=10, H D=$ 11). See text for details about DGX administration. (C-E) Cortical thickness (C), trabecular bone 
452 volume (D) and polar moment of inertia (E) of 16-week-old male WT mice (number of animals: $\mathrm{C}=8$, $453 \mathrm{LD}=10, \mathrm{HD}=11$ ). (F) Serum osteocalcin at 16 weeks of age (number of animals: $C=8, L D=9, H D=$

454 11). One-way ANOVA with Bonferroni post-hoc test was used. ${ }^{*} p<0.05, * * p<0.01, * * * p<0.001$,

$455 * * * * \mathrm{p}<0.0001$. BV/TV = bone volume fraction; $\mathrm{C}=$ control; Cort. $\mathrm{Th}=$ cortical thickness; $\mathrm{HD}=\mathrm{high}$

456 dose; $\mathrm{J}=$ polar moment of inertia; $L D=$ low dose; $O C=$ osteocalcin.

457 Figure 6. Effects of delayed onset or complete suppression of puberty on body and bone length at 458 adult age. (A-C) Nose-to-tail (A), femur (B), and vertebral L5 column (C) length of 16-week-old male 459 WT mice from the indicated groups (number of animals: $C=8, L D=9-10, H D=11$ ). See text for 460 details about DGX administration. (D-E) Serum levels of IGF-1 (D) and IGFBP-3 (E) in 16-week-old 461 mice (number of animals: $C=8, L D=9, H D=11$ ). One-way ANOVA with Bonferroni post-hoc test was 462 used. ${ }^{*} \mathrm{p}<0.05,{ }^{* *} \mathrm{p}<0.01,{ }^{* * * *} \mathrm{p}<0.0001$. $\mathrm{C}=$ control; $\mathrm{HD}=$ high dose; IGF-1 = insulin like growth factor-1; 463 IGFBP-3 = insulin like growth factor binding protein-3; L5 = lumbar 5, LD = low dose.

464 Figure 7. Proposed model for the effects of transient versus persistent sex steroid deficiency 465 induced by chemical castration with GnRH antagonist in male mice. 

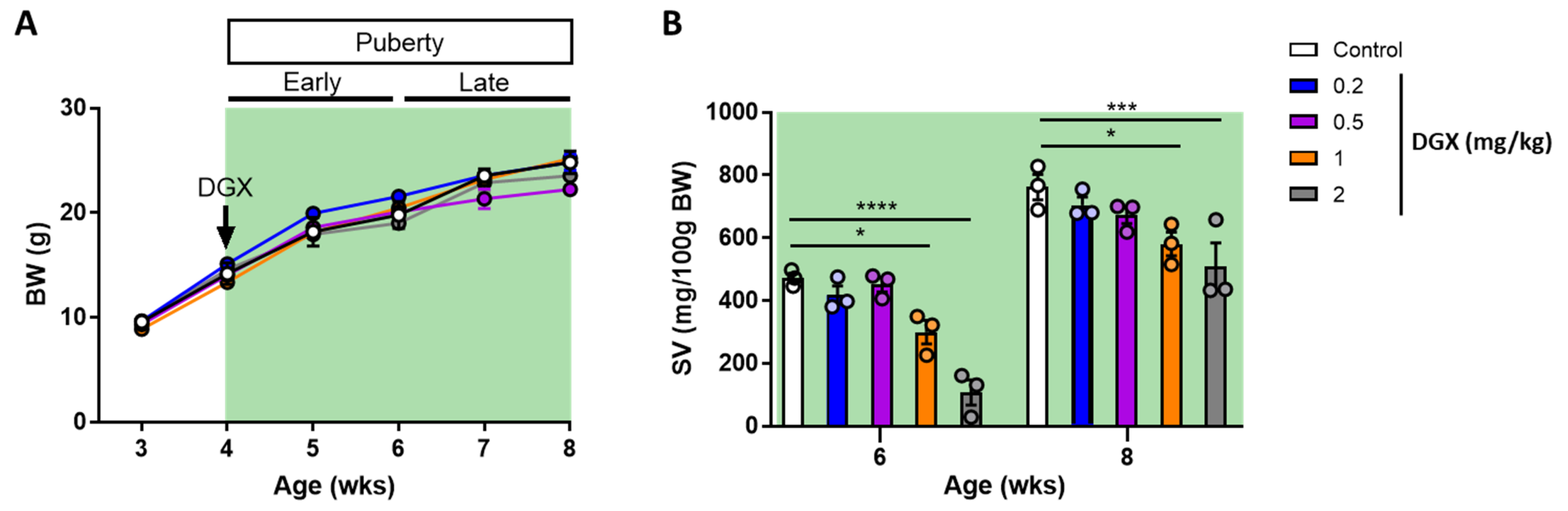
A

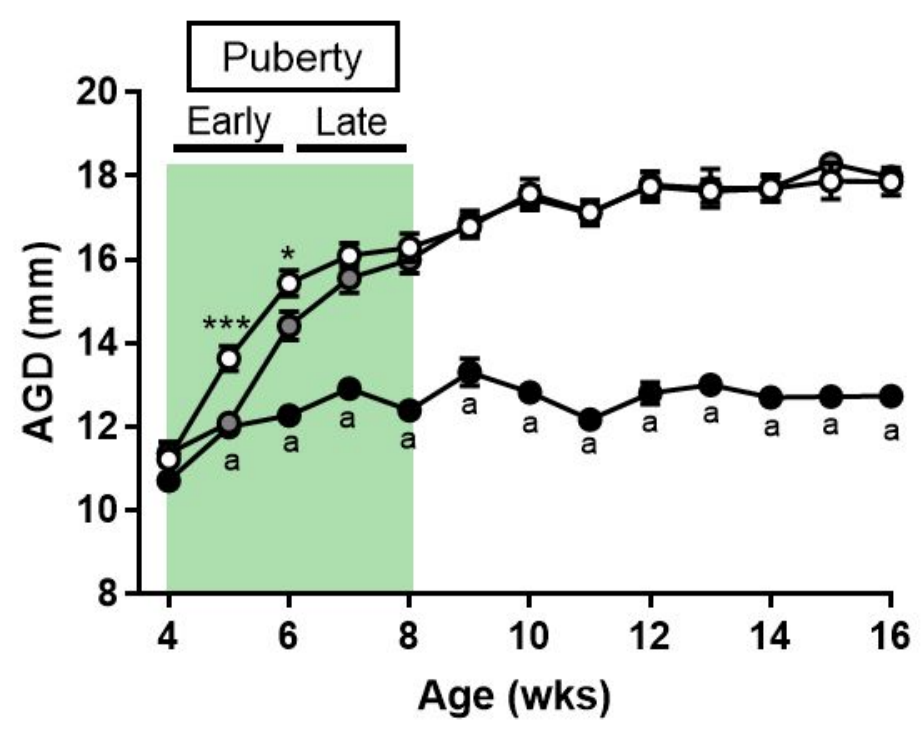

C

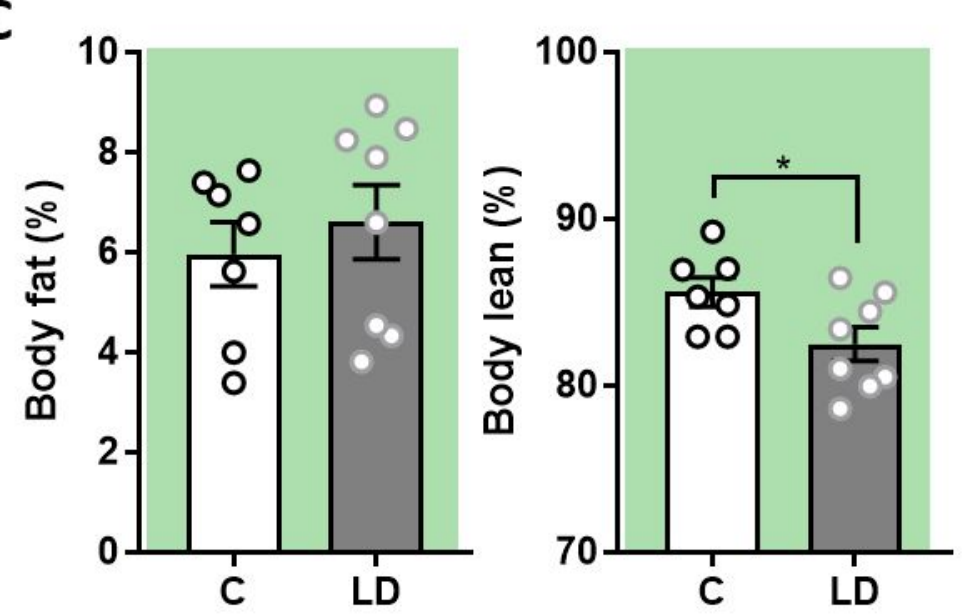

E

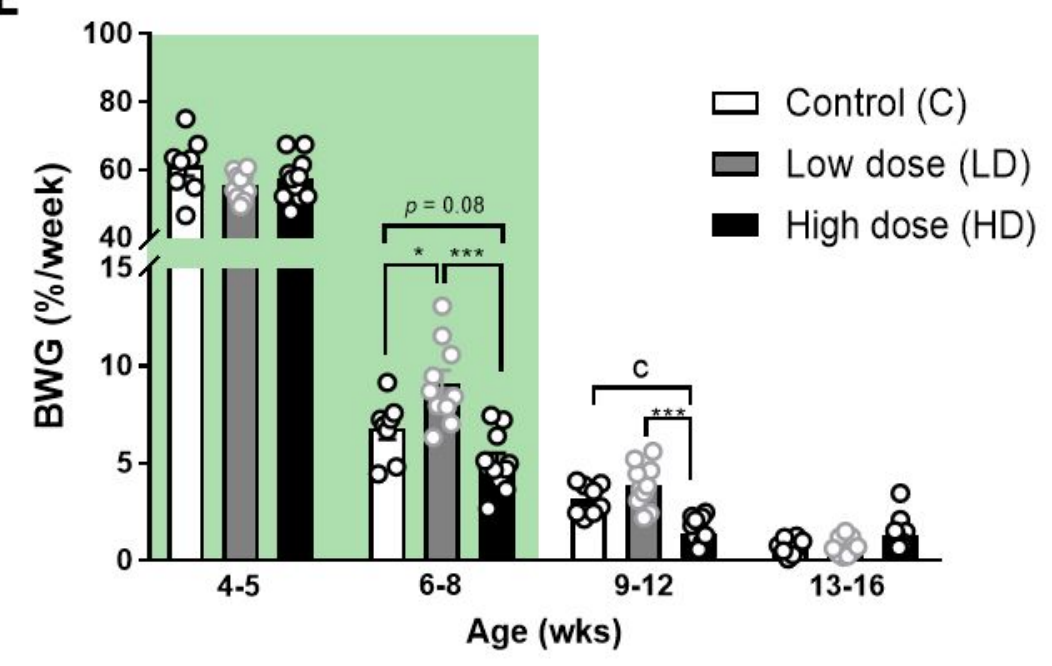

H

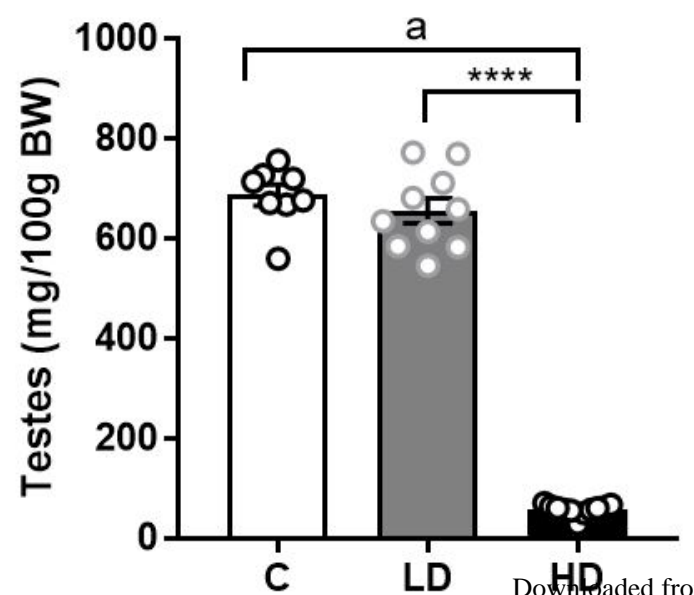

B
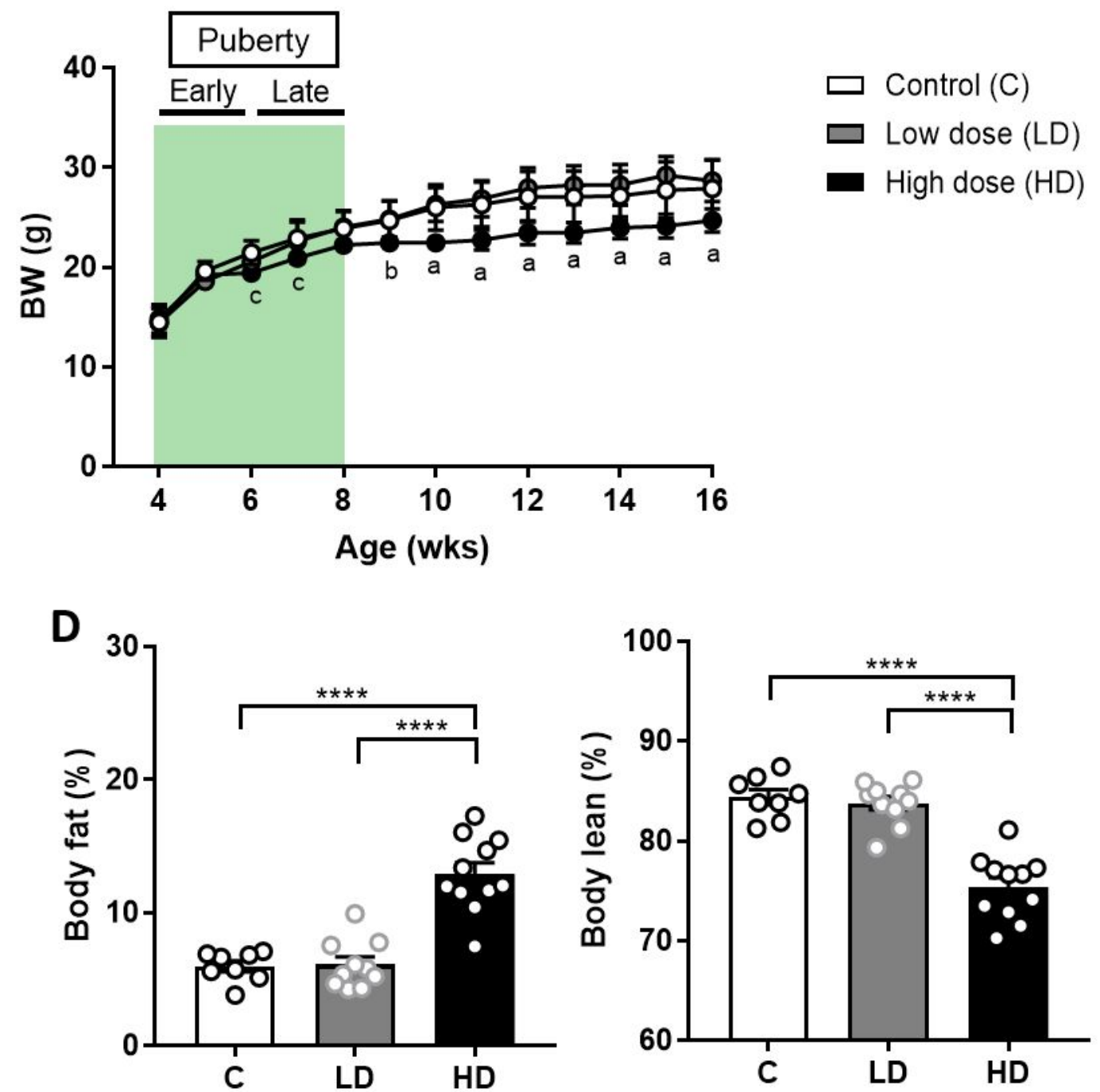

F

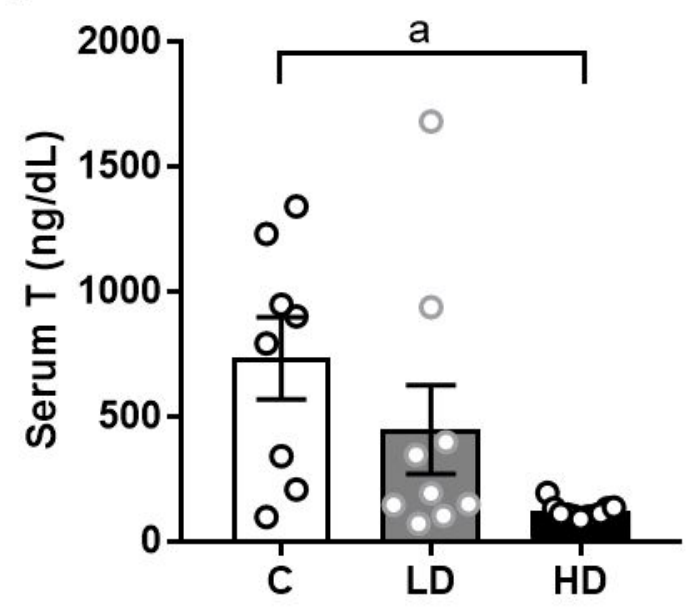

G

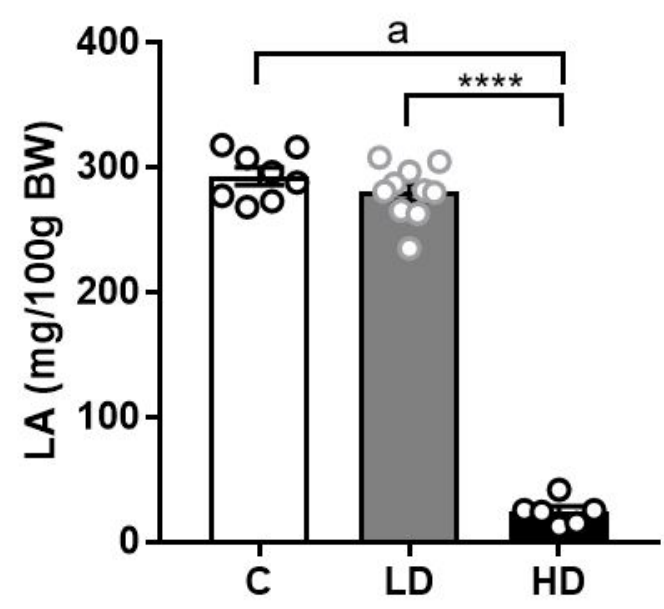

I

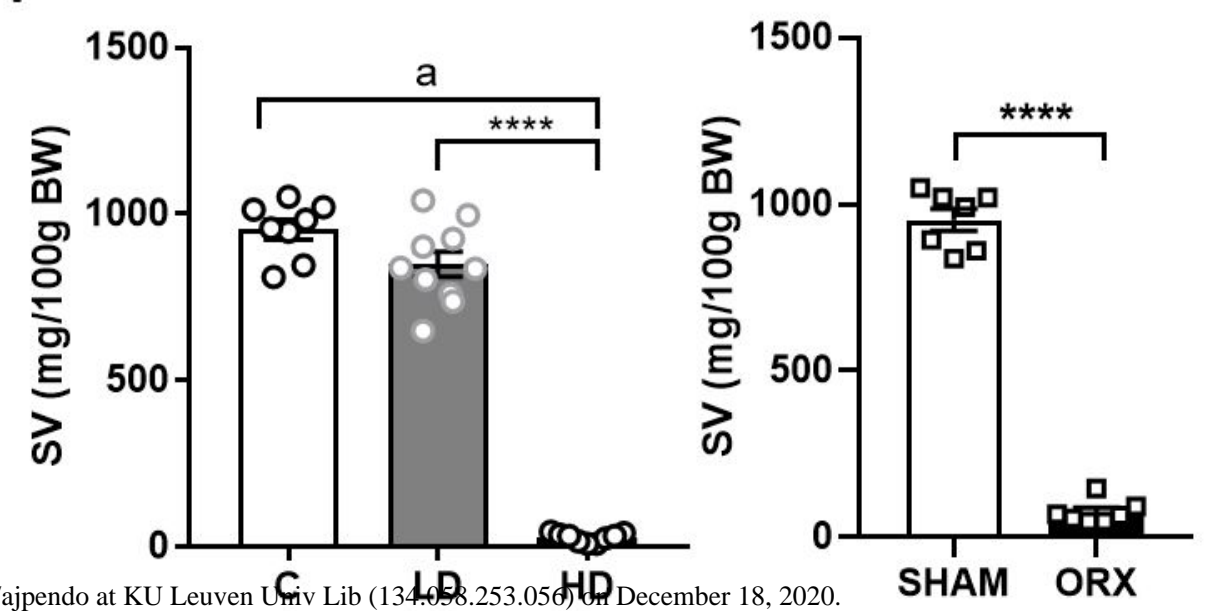


A

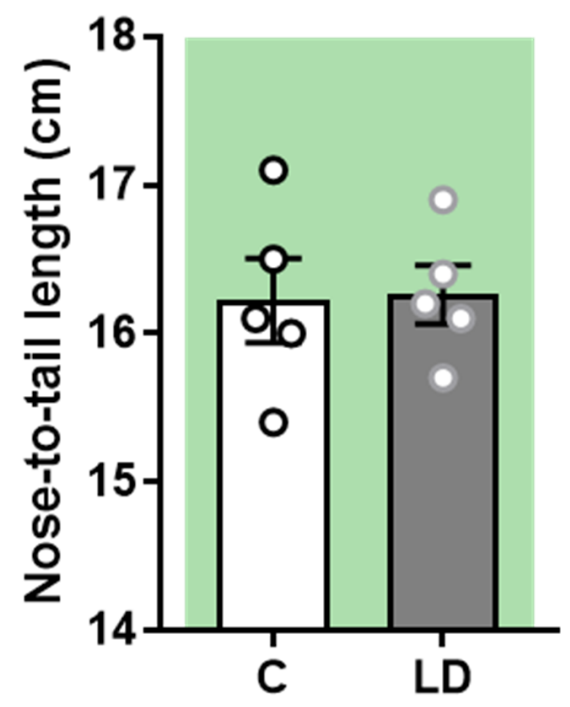

D

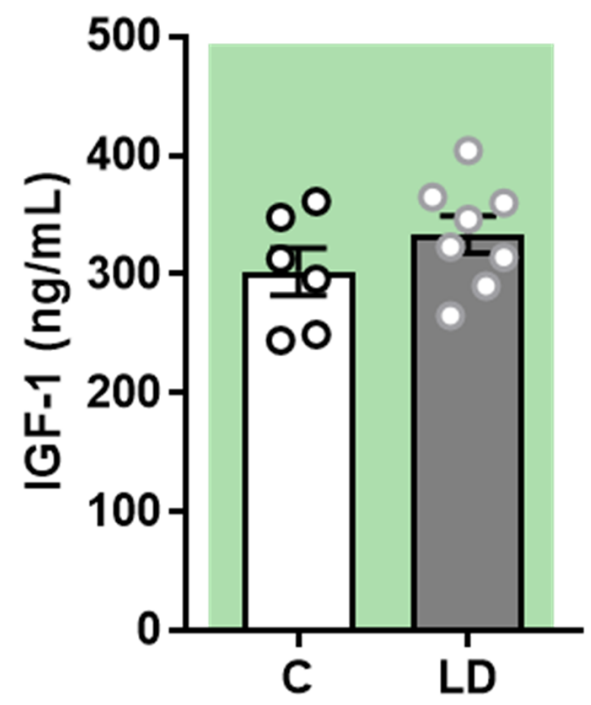

C
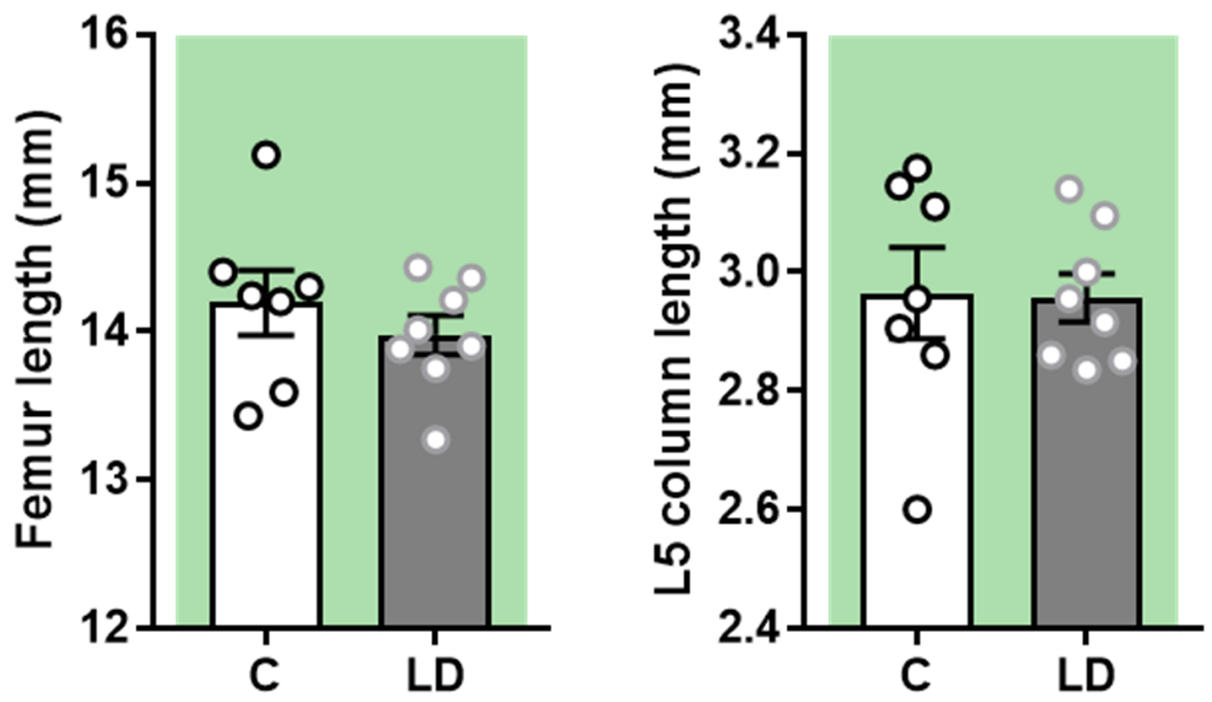

E

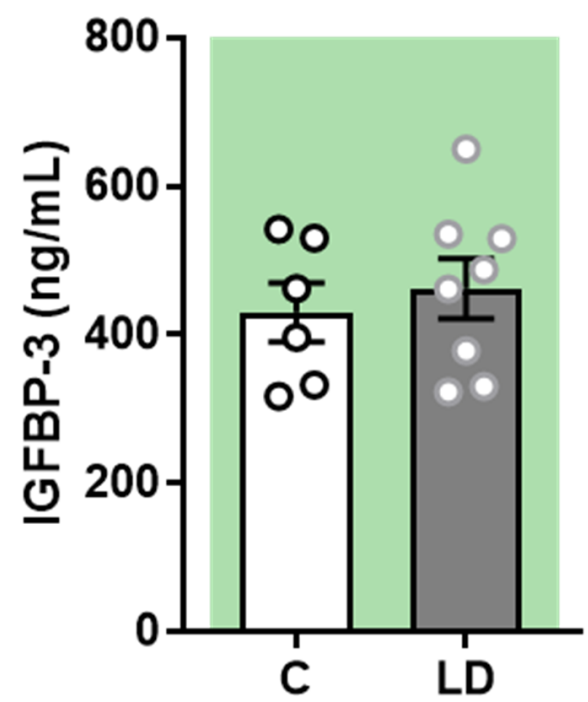



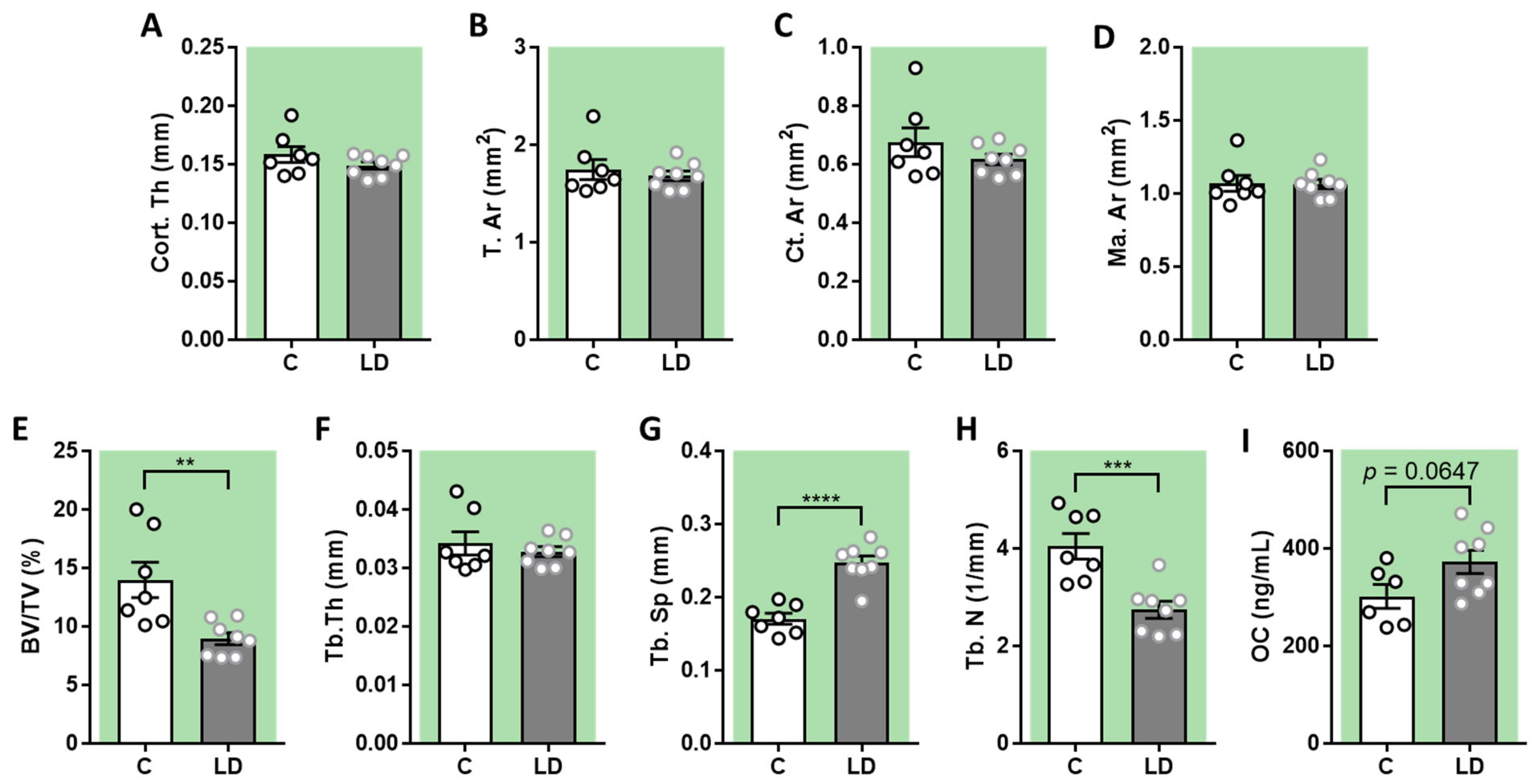
A
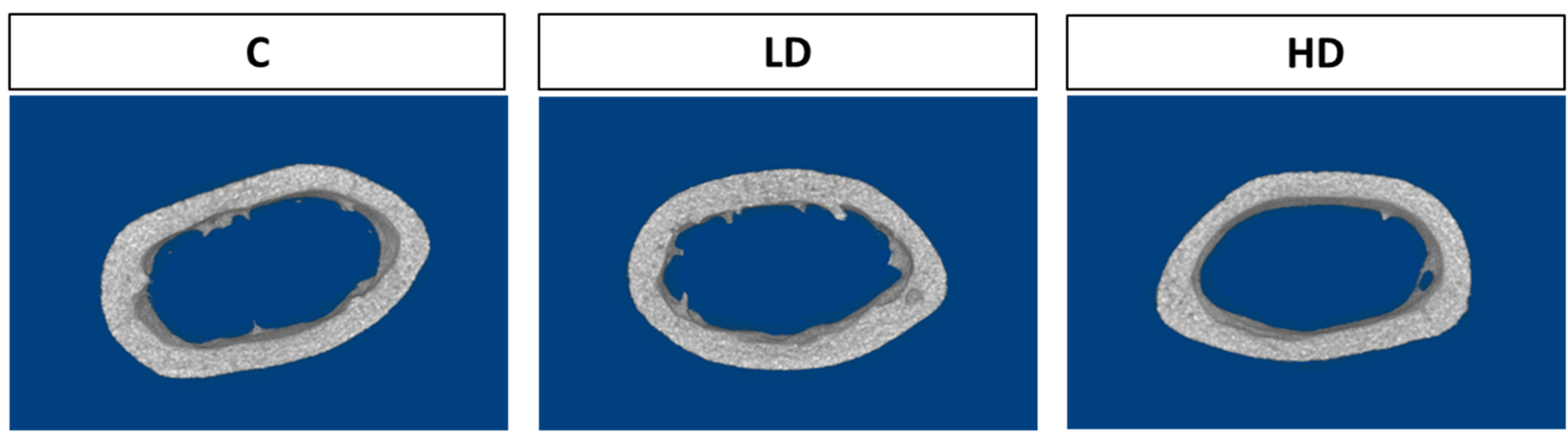

B
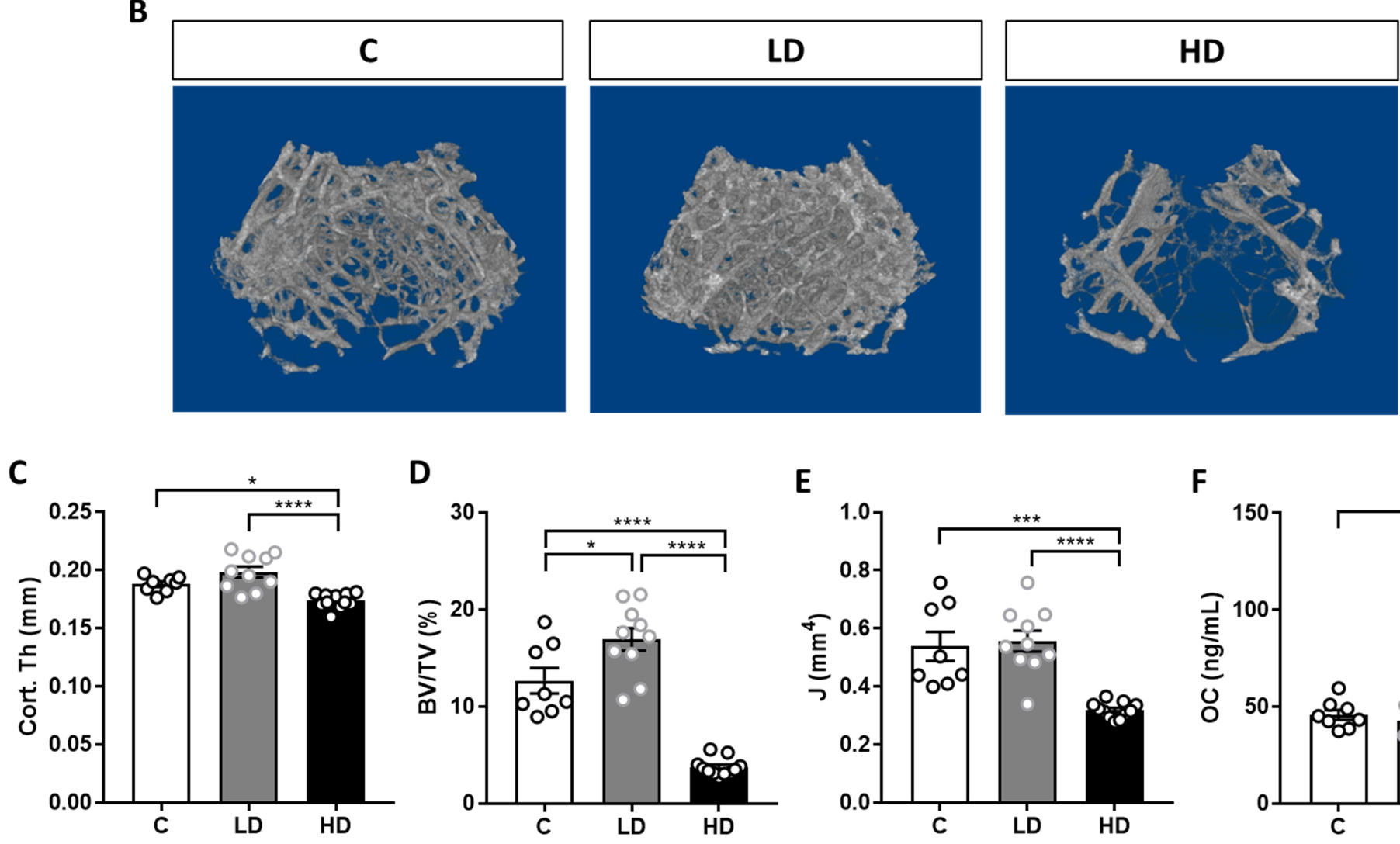

E

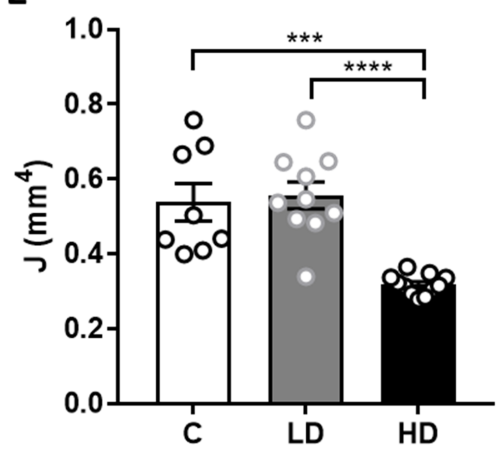

F

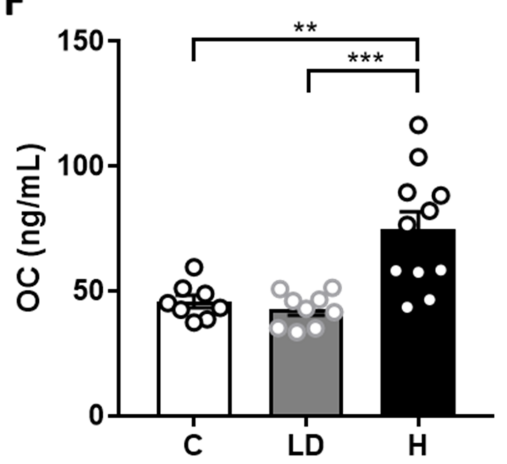



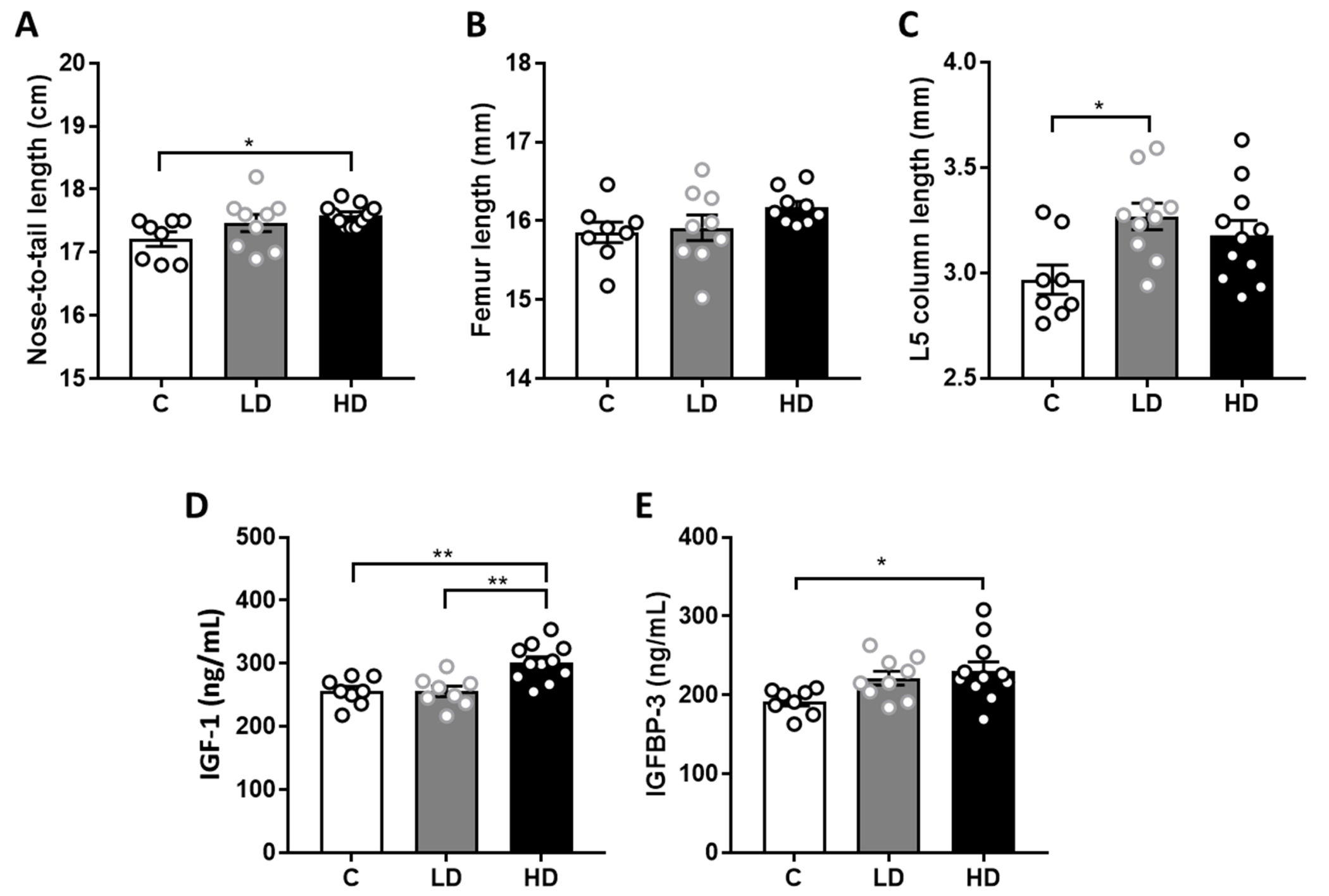


\section{Male mice}

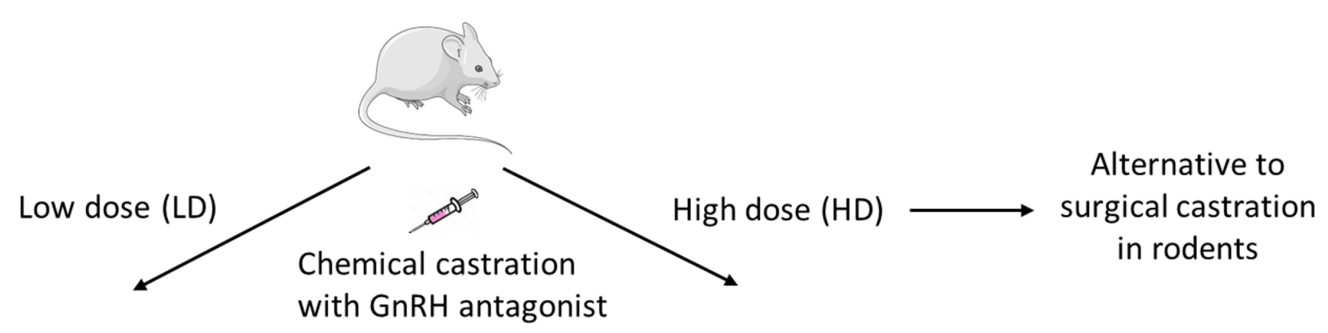

\section{TRANSIENT SEX STEROID DEFICIENCY}

BONE

LINEAR GROWTH

BODY COMPOSITION

CLINICAL RELEVANCE
Trabecular bone loss during puberty but recovery at adult age

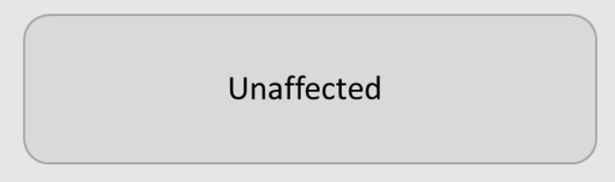

Unaffected

Model for delayed puberty

\section{PERSISTENT SEX STEROID DEFICIENCY}

Trabecular and cortical bone loss

Increased

Fat mass increased Lean mass decreased

Model for hypogonadotropic hypogonadism 
Table 1. Cortical and trabecular bone parameters of femur at adult age. Data were obtained in 16week-old male WT mice and were analyzed with one-way ANOVA with Bonferroni post-hoc test. $\mathrm{Ct} . \mathrm{Ar}=$ cortical area; $\mathrm{Ma} . \mathrm{Ar}=$ medullary area; $\mathrm{Ct} \mathrm{EC}=$ endosteal circumference; $\mathrm{Ct} . \mathrm{PC}=$ periosteal circumference; $\mathrm{Tt} . \mathrm{Ar}=$ total cross-sectional tissue area; $\mathrm{Tb} . \mathrm{N}=$ trabecular number; $\mathrm{Tb} . \mathrm{Sp}=$ trabecular separation; Tb.Th = trabecular thickness.

\begin{tabular}{|c|c|c|c|c|}
\hline & & $\begin{array}{l}C \\
(n=8)\end{array}$ & $\begin{array}{l}\text { LD } \\
(n=10)\end{array}$ & $\begin{array}{l}\mathrm{HD} \\
(\mathrm{n}=11)\end{array}$ \\
\hline \multirow[t]{5}{*}{ Cortical bone } & T. $\operatorname{Ar}\left(\mathrm{mm}^{2}\right)$ & $2.20 \pm 0.11$ & $2.17 \pm 0.08$ & $1.67 \pm 0.02^{* * *}, \# \# \#$ \\
\hline & Ct. $\operatorname{Ar}\left(\mathrm{mm}^{2}\right)$ & $0.90 \pm 0.04$ & $0.95 \pm 0.03$ & $0.71 \pm 0.01^{* * * *}, \# \# \#$ \\
\hline & Ma. $\operatorname{Ar}\left(\mathrm{mm}^{2}\right)$ & $1.26 \pm 0.08$ & $1.22 \pm 0.06$ & $0.96 \pm 0.02^{* *}, \# \#$ \\
\hline & Ct. PC (mm) & $5.81 \pm 0.16$ & $5.81 \pm 0.11$ & $5.07 \pm 0.04^{* * * *}, \# \# \# \#$ \\
\hline & Ct. EC $(\mathrm{mm})$ & $5.68 \pm 0.32$ & $5.50 \pm 0.18$ & $4.35 \pm 0.05^{* * *}$, \#\#\# \\
\hline \multirow[t]{3}{*}{ Trabecular bone } & Tb. Th $(\mu \mathrm{m})$ & $40.53 \pm 0.87$ & $44.33 \pm 1.27^{\mathrm{a}}$ & $33.84 \pm 0.76^{\# \#}$ \\
\hline & Tb. $S p(\mu \mathrm{m})$ & $199.1 \pm 6.37$ & $179.2 \pm 5.61$ & $381.8 \pm 18.33^{* * * *}$, \#\#\#\# \\
\hline & Tb. N (1/mm) & $3.10 \pm 0.28$ & $3.81 \pm 0.24$ & $0.97 \pm 0.08^{* * * *}$ \\
\hline
\end{tabular}

${ }^{a} \mathrm{p}<0.05$, comparison C vs. LD. ${ }^{* *} \mathrm{p}<0.01,{ }^{* * *} \mathrm{p}<0.001,{ }^{* * * *} \mathrm{p}<0.0001$, comparison C vs HD. ${ }^{* \#} \mathrm{p}<0.01$,

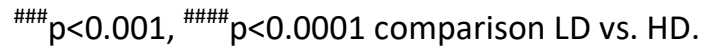




\section{Novel model to study the physiological effects of temporary or prolonged sex steroid deficiency in male mice}

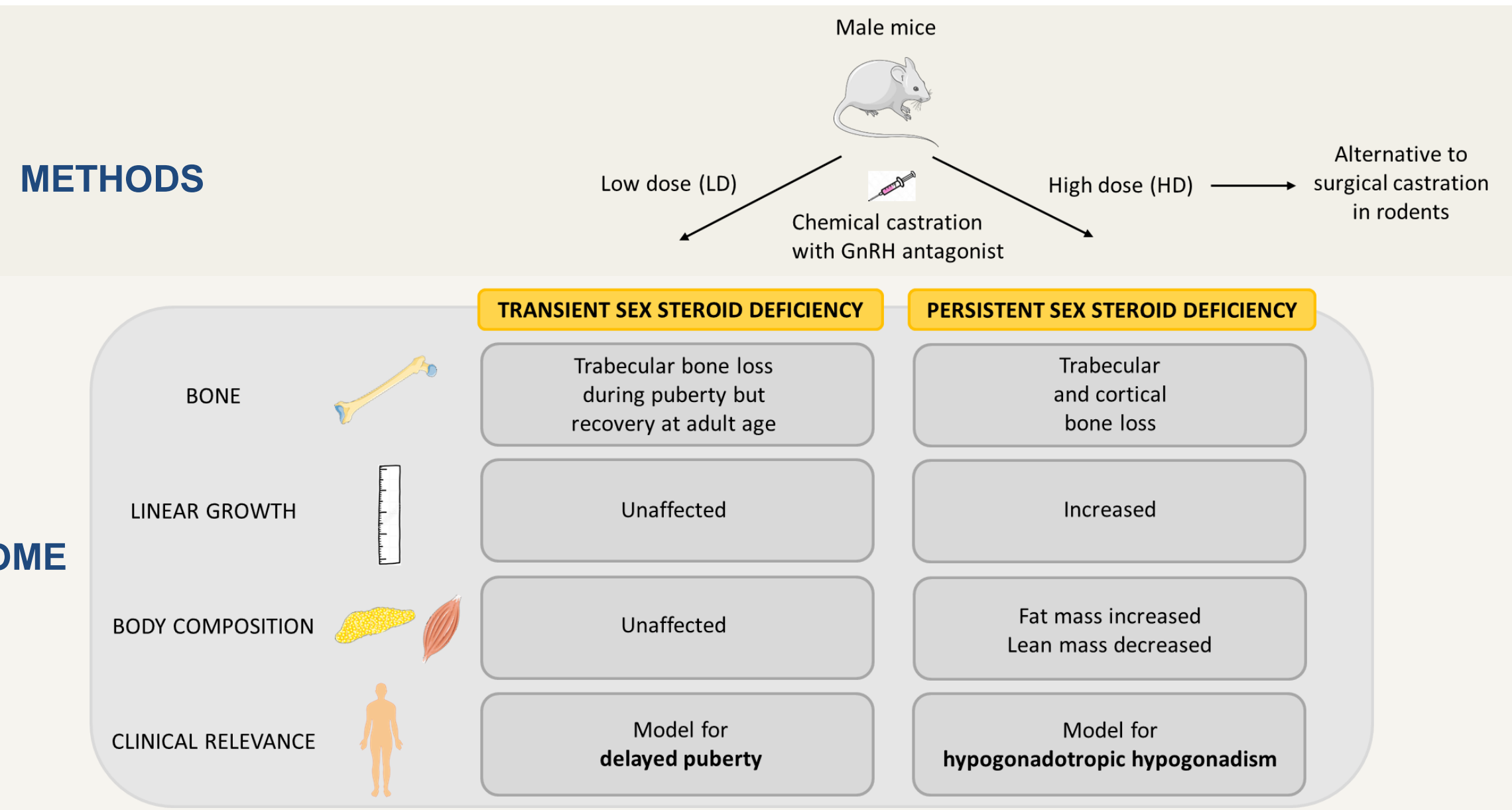

\section{CONCLUSION}

- We developed a rodent model of chemical castration, which can be used as an alternative to surgical castration.

- The transient nature of the intervention enables to study the effects of delayed puberty and

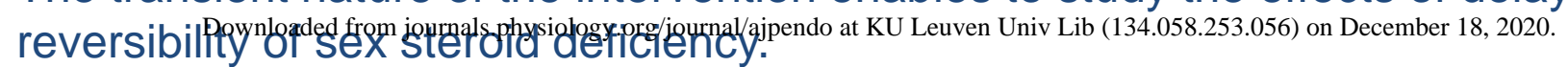

